\title{
DISTRIBUTION OF PHYTOPLANKTON IN THE SOUTHWESTERN AREA OF ALEXANDRIA (ABU-QIR BAY), EGYPT.
}

\author{
Hanan M. Khairy \\ National Institute of Oceanography and fisheries, Alexandria, Egypt. \\ E.Mail address: hanan_khairy@yahoo.com
}

\begin{abstract}
Phytoplankton samples were collected during February, April and June 2004 at fourteen stations. Phytoplankton community represented by 117 species within 66 genus from five groups (Bacillariophyceae; Dinophyceae; Chlorophyceae; Cyanophyceae and Euglenophyceae). There were some fresh, brackish, and marine species. Bacillariophyceae was the most dominant group (71 species) contributing $94.2 \%$ of the total phytoplankton counts. The phytoplankton community is generally more productive at stations 1, 2, 3, 4 and 5 that receiving discharge water from El-Tabia pumping station (Stations 1, 2, 3 and 4) and Lake Edku (St. 5). A gradual decrease was seen in phytoplankton density seawards. The major phytoplankton peak was observed at most stations during June (average $5535 \times 10^{3}$ unit.. $^{-1}$ ). Skeletonema costatum was the most dominant species; it was formed $51.3 \%, 41.3 \%$ and $60.4 \%$ of the total phytoplankton, respectively during February, April and June. The effect of discharge water was more pronounced at St. 4 in front of El-Tabia pumping station. Species diversity index displayed narrow temporal and spatial variations from 1.02 to 2.85 nats. Throughout the bay, station 3 sustained the lowest diversity index (1.38 nats), while station 14 recorded the highest value (1.95 nats).
\end{abstract}

Keywords: Chlorophyll-a- Diversity- Eutrophication- Phytoplankton- Skeletonema costatum.

\section{Introduction}

In the Mediterranean Sea, eutrophication appears to be limited mainly to specific coastal and adjacent offshore areas. Several and sometimes severe cases of eutrophication are evident, especially in enclosed or semi-enclosed bays which receive elevated nutrient loads from agricultural, together with direct discharges of untreated or poorly treated domestic and industrial wastewaters (Richardson and Jorgensen, 1996). Abu-Qir Bay is one of the most interesting bays along the Egyptian coast; it is a semicircular basin in the Mediterranean coast of Egypt, lying between longitudes $30^{\circ} 4^{\prime}$ and $30^{\circ} 21^{\prime} \mathrm{E}$ and latitude $31^{\circ} 16^{\prime}$ and $31^{\circ} 30^{\prime} \mathrm{N}$. The Bay is about $360 \mathrm{Km}^{2}$ and the water volume is $4.3 \mathrm{Km}^{3}$ (Said et al., 1995). It has a shoreline of about $50 \mathrm{Km}$ between Abu-Qir peninsula at the west and the Rosetta branch of the River Nile at the east and maximum depth of about $16 \mathrm{~m}$. It receives the effluents of many industrial activities as food processing, refineries, fertilizers, paper mill power station, domestic sewage and agricultural drainage 
(Said, 1991; Tayel, 1992). These wastes are collected and dumped into the bay through El-Tabia Pumping station (TPS) situated at the southern extremity of the bay. The daily discharge of the combined liquid wastes is estimated to be about $2 \times 10^{6} \mathrm{~m}^{3}$. The bay receives also agricultural drainage water from Lake Edku through Boughaz El-Maadiya (Mohamed and El-Maradny, 2001). The discharge water caused continuous changes in the water characteristics, enhancing its fertility. Therefore, the bay as one of the most productive fishery grounds along the Mediterranean Coast of Egypt. Many investigations were done in the bay including hydrographic conditions, pollution problems (El-Deeb, 1977; Anonymous, 1984; Tayel, 1992; El-Rayes et al., 1993; Fahmy, 1997; Mohamed and El-Maradny, 2001; Abdel-Aziz et al,. 2001; Aly-Eldeen, 2006). However, a few studies were conducted on phytoplankton in Abu-Qir Bay (Dowidar et al., 1983; Samaan and Mikhail, 1990; El-Sherif and Gharib, 1994; El-Sherif and Mikhail, 2003; Shams-El-Din and Dorgham, 2007). Because of the effluents discharged into the bay causes continuous changes in its ecological characteristics, these changes affect the biological components of the bay's ecosystem. Since 2000, no studies were carried out on phytoplankton community in Abu-Qir Bay. Thus, the objective of this study is to study the dynamics of the phytoplankton in Abu-Qir Bay to analyze the changes in the phytoplankton composition, abundance and biomass, and to establish which of environmental factors determine these dynamics. Comparing the present phytoplankton composition of Abu-Qir Bay with the previous studies will also give.

\section{Material and methods}

Abu-Qir Bay lies at the southwestern area of Alexandria and is considered one of the main fishery basins. It is continuously facing pollution problems, however; it receives the effluents from many sources (industrial activities; domestic sewage and agricultural drainage). The western part of AbuQir Bay was influenced by the wastewater from El-Tabia pumping station (TPS) and brackish water from Lake-Edku (Fig. 1). This work is a part of the research plan of Marine Environment Division of the National Institute of Oceanography and Fisheries entitled "Effect of land based sources on physical, chemical and biological characteristics of Abu-Qir Bay waters". Sampling was carried out during 2004, including three months (February, April and June) representing three seasons (winter, spring and summer). Fourteen stations were chosen representing different ecological conditions (Figure 1). The data for temperature, salinity and dissolved oxygen were attained from the plan. One liter of water samples were collected by using Ruttener bottle sampler for estimation the phytoplankton abundance. The phytoplankton samples were preserved immediately with $4 \%$ neutralized formalin. Estimation of the phytoplankton abundance was carried out by the sedimentation method (Utermöhl, 1958) and the results expressed as unit per liter (the unit-comprised cells, colonies and filaments). For the identification 
of the phytoplankton species the following works were consulted (Balech, 1948 and 1980; Pankow, 1976; Krammer and Lange-Bertalot, 1986; Heimann, et al., 1995).

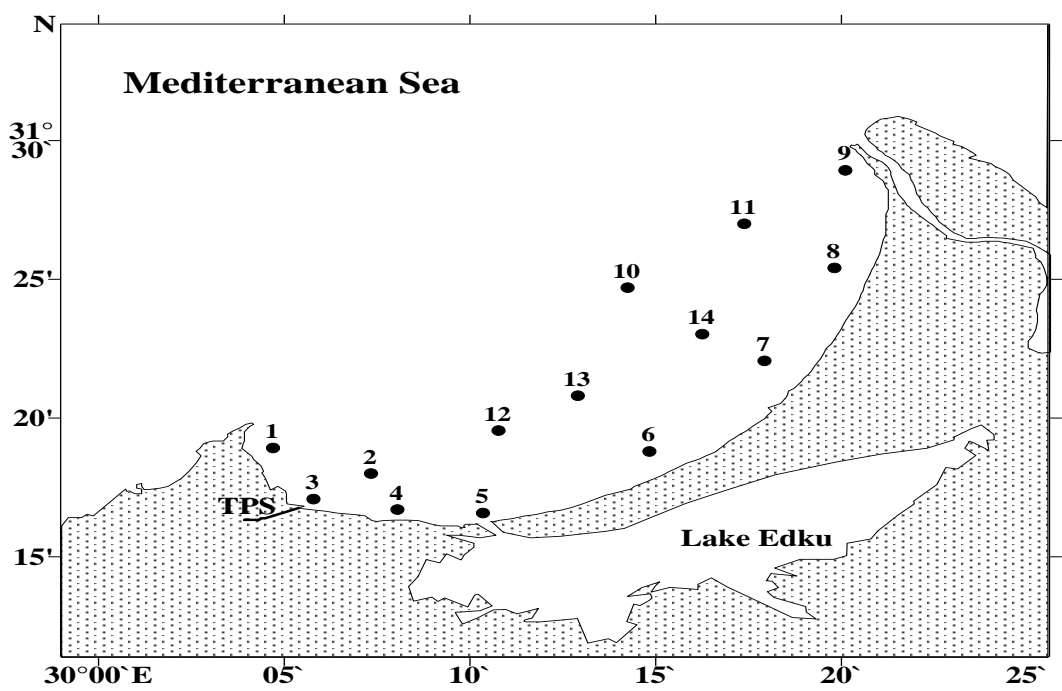

Figure (1): Map of Abu-Qir Bay area and location of the sampling stations

Species diversity index was estimated according to the equation of Shannon and Weaver (1963) the results was expressed as nats.

$$
\mathrm{H}=-\sum_{\mathrm{i}=1} \mathrm{Pi} \operatorname{Ln} \mathrm{Pi}
$$

Where $\mathrm{Pi}=$ importance probability for each species $(\mathrm{n} / \mathrm{N}$ is the proportional of $\mathrm{i}$, the ni species) to the total number of phytoplankton cells $(\mathrm{N})$.

Correlation coefficient as well as stepwise multiple regression equations by using Minitab program at a confidence limit $95 \%(\mathrm{P} \leq 0.05$, ) were evaluated $(n=41)$ to quantize the phytoplankton abundance in relation to the most correlative parameters.

\section{Results}

Changes in temperature at different sampling stations (Sts) in Abu-Qir Bay are shown in Figure (2). The variations of the surface water temperature of the bay are very closely related to those of air temperature. During February, the lowest values of air temperature were observed, the surface water temperature varied from $16.2^{\circ} \mathrm{C}$ at Sts. 8 and 9 to $18.3^{\circ} \mathrm{C}$ at St. 1 . In April, the water temperature increased westward from $19.6^{\circ} \mathrm{C}$ at St. 12 to $22.8^{\circ} \mathrm{C}$ near TPS (St. 4). During June, the air temperature reached its maximum value throughout the year, while the surface water temperature varied between $26.0^{\circ} \mathrm{C}$ at St. 9 and $31.9^{\circ} \mathrm{C}$ at 
St. 13. Strong positive correlation exists between total phytoplankton and water temperature $(\mathrm{r}=+0.61, \geq 0.05)$.
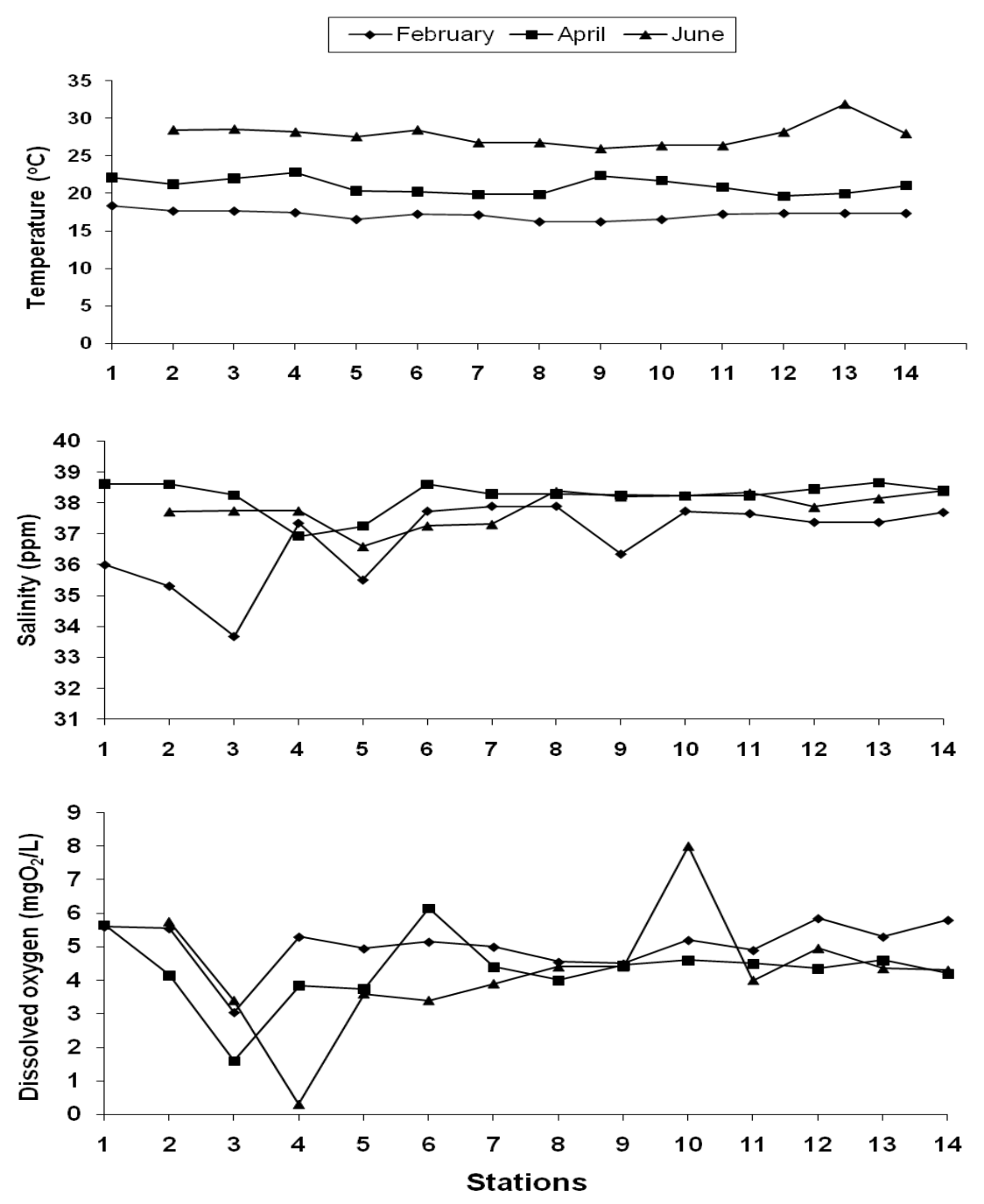

Figure (2): Surface water temperature, salinity and dissolved oxygen at different sampling stations at Abu-Qir Bay during February, April and June 2004

The surface water salinity in Abu-Qir Bay (Figure 2) varied locally within a wide range. During February, the minimum water salinity 33.67 ppt was recorded at TPS (St. 3), due to the influence of the brackish water from TPS. The salinity values of less than $37.00 \mathrm{ppt}$ were found in the western part of Abu-Qir Bay. In April, due to a decrease in the amount of fresh water discharged from the river as compared to winter month, the surface salinity increased from $36.92 \mathrm{ppt}$ 
at St. 4 to $38.65 \mathrm{ppt}$ at St. 13. During June, the surface salinity varied between $36.58 \mathrm{ppt}$ at St. 5 and $38.38 \mathrm{ppt}$ at St. 14. It was due to the effect of the brackish water from Lake Idku and the waste water from TPS.

Regarding with dissolved oxygen (DO) concentrations (Figure 2), the values in February ranged between $3.05 \mathrm{mgO}_{2} \cdot \mathrm{L}^{-1}$ (St. 3) and $5.85 \mathrm{mgO}_{2} \cdot \mathrm{L}^{-1}$ (St. 12). In April, between $1.60 \mathrm{mg} \mathrm{O}_{2} \cdot \mathrm{L}^{-1}$ at TPS and $6.15 \mathrm{mg} \mathrm{O}_{2} \mathrm{~L}^{-1}$ at St. 12 , while during June, it ranged from $0.30 \mathrm{mg} \mathrm{O} \mathrm{L}^{-1}$ near TPS (St. 4) to $5.75 \mathrm{mg} \mathrm{O}_{2} \mathrm{~L}^{-1}$ (St. 2), station 4 sustained the lowest values throughout the year.

The phytoplankton community in Abu-Qir Bay comprised 117 species within 66 genera (Table 1). Most of the recorded species are eurythermal, appeared during the study period, also euryhaline tolerate a wide range of salinity, but low numbers exist at high salinity. Five algal groups were represented at the study area, namely, Bacillariophyceae (71 species); Dinophyceae (18 species); Chlorophyceae (12 species); Cyanophyceae (12 species) and Euglenophyceae (4 species). As it is usually found in semi-enclosed regions affected by discharged freshwater; Bacillariophyceae appeared to be the most dominant group qualitatively and quantitatively. Qualitatively, it represented by 71 species with 41 genera, while quantitatively was formed with average of $94.2 \%$ of the total phytoplankton count (Table 2). Dinophyceae showed only in 9 genera (Table 1). The other fresh water groups showed remarkable by low numbers of genera with low counts and its occurrence may be coming from Lake Edku. They were represented by 6 genera for Chlorophyceae, 9 for Cyanophyceae and one genus for Euglenophyceae (Table 1).

The appearance of different species revealed that, 117 species were observed throughout the whole area (Table 1). During February, the higher species number were represented at St. 14 (72 species), while the lowest number (40 species) were observed at St. 7. On the other hand, during April, the higher species number (78 species) was recorded at St. 4, while the lower numbers (40 species) were observed at St 14. During June, the higher species number (76 species) were recorded at St. 5, but the lowest one (49 species) were observed at St. 4.

Regarding with the number of species during different months (Table 1), February showed the higher species number (112 species), while April attained the lower number of species (99 species), on the other hand, June have 104 species.

Also, according to the total phytoplankton counts distribution, the western part was the most productive stations (Sts.1, 2, 3, 4 and 5) in the bay (Table 3). On the other hand, the other stations showed pronounced low averages. The predominant species which showed strongly distribution during the study period along the sampled stations were Skeletonema costatum; Nitzchia spp; Rhizosolenia delecatula; and Asterionella spp. 


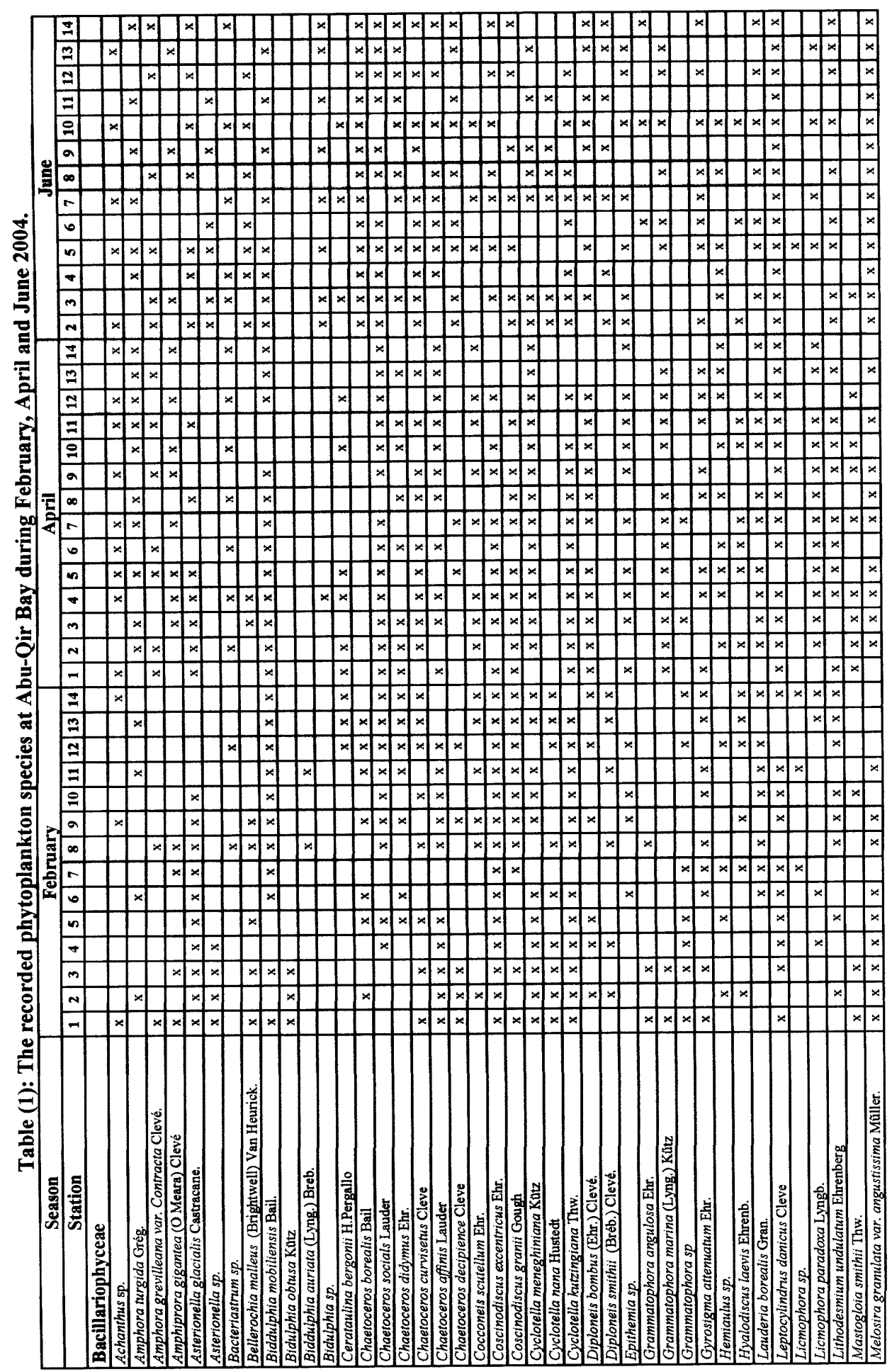


Distribution of Phytoplankton in the Southwestern area of Alexandria (Abu-Qir Bay), Egypt.

\begin{tabular}{|c|c|c|c|c|c|c|c|c|c|c|c|c|c|c|c|c|c|c|c|c|c|c|c|c|c|c|c|c|c|}
\hline & $x \times$ & & & & & $x$ & & & $x \times$ & $x$ & & & & $x$ & $\times$ & & & $\star x$ & & & 9 & $\times$ & & & $\times \times \times$ & & & \begin{tabular}{|l|l|}
$\mid x$ & \\
\end{tabular} & \\
\hline & & & 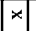 & 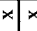 & & $x$ & & & $\nexists \times$ & $x$ & & & $\times$ & × & $x \neq$ & & & $\times$ & & & ना & $|\times|$ & & $\bar{x}$ & $x$ & & & 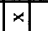 & $\mid x$ \\
\hline & $x \times$ & \begin{tabular}{|l|l}
$x$ & \\
\end{tabular} & $x$ & & $x$ & $x$ & & $\star x$ & $\bar{x}$ & $x$ & & & $\times$ & & & & & & $x=$ & & ले & $|\times|$ & & & $x \times x$ & & $\bar{x} x$ & $x \mid x$ & \\
\hline & $\times$ & & & $\times x$ & & $x$ & & & $\times x$ & $\times$ & & ^ & $\times$ & $\bar{x} \times$ & $x \times$ & & & $\times x$ & $\times$ & $\bar{x}$ & ত্ল & $\bar{x}$ & & $\bar{x}$ & \begin{tabular}{l|l}
$x$ & $x$
\end{tabular} & & & $|x| x$ & $x \times$ \\
\hline & $x \times$ & $x$ & & & $x$ & $x$ & $\times$ & & $\times x$ & $\star x$ & & & $\times$ & $\times$ & & $x \times$ & & & $\approx$ & & 7 & $\times$ & & & \begin{tabular}{c|c|}
$x$ & $x$
\end{tabular} & & $\star x$ & & \\
\hline & $\times$ & & $x$ & $x$ & & $x$ & & & $x$ & $x$ & & $\bar{x}$ & $\times$ & $x$ & $\times$ & & & $\bar{x}$ & & & $\vec{N}$ & & & & $x \times x$ & & & $|x| x$ & $x$ \\
\hline & $x \times$ & . & & $\bar{x}$ & $x$ & 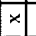 & & & $\star x \times$ & $x$ & & $\bar{\star}$ & $\times$ & $\bar{x} \times$ & & & & 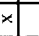 & $x$ & $x$ & 官 & & $x$ & & $x \times x$ & & $\bar{x}$ & 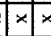 & 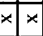 \\
\hline & $x \times$ & & $|x|$ & $\times$ & & $\star x$ & & & $7 x$ & $\star$ & & $\bar{x}$ & $x$ & $\bar{x}$ & & $x \times$ & & $x \mid x$ & & & 戹 & $\times$ & & & \begin{tabular}{c|c|}
$x$ \\
\end{tabular} & & $x \times$ & & \\
\hline & $x \times$ & x & & & & $\times$ & & & $\times$ & $x$ & & $\bar{\star}$ & \begin{tabular}{|l|}
$\times$ \\
\end{tabular} & $\bar{x}$ & 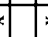 & $\times$ & & $\star x$ & $|\times|$ & & 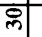 & $x$ & & & $\times \times \mid x$ & $x$ & & $|x| x$ & 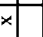 \\
\hline & $x$ & \begin{tabular}{l|l}
$\bar{x}$ \\
\end{tabular} & $x$ & $x x$ & $x$ & $x$ & & & $\times$ & $x$ & & $\bar{\star}$ & $\times$ & & & & & $\bar{x}$ & & & 7 & $\times$ & $x \times$ & & \begin{tabular}{l|l}
$x$ & $x$
\end{tabular} & & & $x \times x$ & 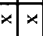 \\
\hline & $x \times$ & & $\times$ & & $\star$ & $x$ & $\left.\right|^{\star}$ & & $\star \star x$ & $x$ & & & \begin{tabular}{|l|} 
\\
\end{tabular} & & & $x \times$ & & $\bar{x}$ & $\times$ & & ले & & & & $x \times x$ & & $x \sqrt{x}$ & $\mid x$ & \\
\hline & T & \begin{tabular}{|l|} 
\\
\end{tabular} & & $x$ & & $x$ & & & $\times \times$ & $\times$ & & & $\times$ & $x \times$ & $x \times$ & & & & $\star x$ & & क्ले & & $x$ & & \begin{tabular}{c|cc}
$x$ & $x$
\end{tabular} & $x \times$ & & 7 & $*$ \\
\hline & $\times \times$ & $\bar{x}$ & & $\times$ & 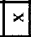 & $x$ & & & $\times x$ & $\times$ & & & $\times$ & . & $x \times$ & $x \times$ & & $x \times$ & $\times$ & $\times$ & $\bar{\nabla}$ & $x$ & & & $\times \times \times$ & & $x$ & $|x|$ & \\
\hline & $x$ & $x$ & & & & $x$ & x & & 7 & & & & $\times$ & $x$ & & & & $\bar{x}$ & $x$ & & $\pi$ & & $\star$ & & $x \times x$ & $x \times$ & 7 & $x$ & \\
\hline & $x \times$ & & & & 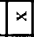 & $x$ & & & $x$ & 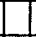 & & & $x$ & & & $x$ & & $x$ & $x$ & $x|x|$ & $\sqrt{-1}$ & & $x$ & & $\times \times$ & & $\times$ & $x$ & $x$ \\
\hline & $\times \mid$ & & $|x|$ & & & $x$ & $x$ & & $\times$ & $\star x$ & & $x$ & $x$ & $\times \times$ & & $x$ & & $x$ & & $\times 1$ & 杹 & & $\times$ & & $\times|\times| x$ & $x \times 1$ & & $x \mid$ & \\
\hline & $\times \times$ & & $x$ & & $x$ & $\nexists$ & & & d & 10 & 7 & & $x$ & & $\times$ & & & $x \times$ & $x$ & & 色 & & $\times$ & & $x \times$ & & $\star$ & $x \mid x$ & $x \times$ \\
\hline & $x$ & $\stackrel{\star}{*}$ & & & & \begin{tabular}{|l|}
$x$ \\
\end{tabular} & & & 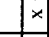 & $\times$ & & $\times$ & $x$ & 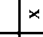 & & $x \mid x$ & & $x \times$ & $x$ & & 总 & & $\times$ & \begin{tabular}{|l|l|}
$x$ & $\times$ \\
\end{tabular} & \begin{tabular}{l|l}
$x$ & $x$ \\
\end{tabular} & $x \times$ & & $x$ & $x$ \\
\hline & $x \times \mid$ & & & $x$ & $\approx$ & & $x$ & & $x$ & & 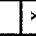 & & $x$ & $x$ & $\times$ & & & $\times$ & & & & & & & $x \times$ & & $\times$ & $x \mid x$ & $x \times$ \\
\hline & $x|x|$ & $\star$ & & & & $x$ & & $\star x$ & $\times x$ & $x$ & & $\approx$ & $x$ & $x$ & & $x$ & & 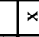 & & & ले & & \pm & & $x \times x$ & $x \times$ & & $\times$ & \\
\hline & $\nexists$ & & & & $x$ & & $x$ & & $x$ & $x$ & $x$ & & $x$ & & $\times$ & & & $\times$ & $x$ & & के & & & & $x \times \times$ & & $x$ & $\times x$ & $x \times$ \\
\hline & $x \times$ & $x$ & & & & $\star x$ & & & $\star x$ & & & $\star$ & $x$ & * & & F & & $x \times$ & $x$ & $x \times \mid$ & ल्ल & $x$ & & & & $x \times$ & & $x \mid$ & \\
\hline & $x \times$ & & $x$ & $\star$ & $\star$ & 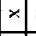 & & $x$ & $\times x$ & $x$ & $\times$ & & $x$ & $*$ & $\times$ & $x \times$ & & $x$ & $|x|$ & $\times \times \mid$ & T: & & 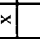 & & $x \mid x$ & $x$ & & $x$ & \\
\hline & $x \times 1$ & $x$ & & 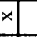 & $x$ & $x$ & $x$ & & $7 x$ & $x$ & $x$ & 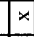 & $x$ & $\bar{x}$ & & $\star$ & $x$ & $x \times$ & $x$ & & ; & & & & $\times \times \times$ & $x \times$ & & $x \mid x$ & \\
\hline & $\star x$ & $x$ & & & $x$ & $\star$ & $x$ & & $\star$ & $x$ & & & $x$ & 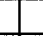 & & $x$ & $x$ & $x \times$ & & $\times$ & के & $x$ & & & $x \times x$ & & x. & $x \mid x$ & $x$ \\
\hline & $\times x$ & $x$ & & $\times$ & $x$ & $x$ & $x$ & $x$ & $x$ & & $\times x$ & $x$ & $x$ & $\times x$ & & $x$ & & \begin{tabular}{l|l}
$* x$ &
\end{tabular} & $|x|$ & & 8 & & $*$ & & \begin{tabular}{l|l}
$x \times$ & $\times$
\end{tabular} & & \begin{tabular}{l|l}
$x$ \\
\end{tabular} & $|x| x$ & \begin{tabular}{|c|}
$x$ \\
\end{tabular} \\
\hline & $\times \times$ & $x$ & & $x$ & & $\times$ & & $x$ & $x$ & $x$ & $\bar{x}$ & $x$ & $x$ & & & $x \times$ & $x$ & $\times x$ & & & 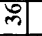 & & $\star$ & & $x \mid x$ & $x \times$ & & $|x| x$ & \\
\hline & $x$ & $x$ & & $x$ & & $x$ & $\sqrt{x}$ & & $x$ & & $\bar{x}$ & $x$ & प & $\bar{x} x$ & & $x$ & & $\bar{x}$ & & & \begin{tabular}{|l|l|l|} 
& \\
\end{tabular} & $x$ & $\bar{x}$ & \begin{tabular}{|l|l|}
$x$ & \\
\end{tabular} & \begin{tabular}{l|l}
$x \times$ \\
\end{tabular} & $x \times$ & $x \sqrt{x}$ & $x=x$ & 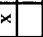 \\
\hline & & $x$ & $\times$ & $\bar{x}$ & $x$ & & $x$ & & $x$ & $x$ & $\times 1$ & $x$ & $\times$ & 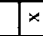 & & $\star$ & & $\bar{x}$ & \begin{tabular}{|l|}
$x$ \\
\end{tabular} & & m & $|\times|$ & $x$ & $|x| x$ & $x \times x$ & $x$ & & $x$ & \\
\hline & $x \times$ & $\bar{x}$ & $x$ & $\bar{x} x$ & $x$ & $x$ & $x$ & & $x$ & 1 & 7 & $x$ & 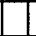 & $\bar{x}$ & & 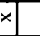 & $x$ & $\bar{x}$ & $|x|$ & & F & 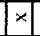 & & $|x| x$ & $x \times x$ & $x$ & 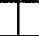 & $\times \mid$ & \\
\hline & $x$ & $\bar{x}$ & $\times$ & $x$ & & & 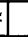 & & $\times x$ & & $\times x$ & $x$ & & $\star$ & & $x$ & & $\bar{x}$ & 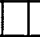 & & न & $\times$ & $\bar{x}$ & $|x| x$ & $x \times \mid \times$ & $x$ & & $x$ & \\
\hline & 0 & $\times x$ & $\times$ & $x$ & $x$ & & Ix & & $x$ & $x$ & $\times 1$ & & $x$ & $x \bar{x}$ & & $x$ & & $x$ & $x$ & & 吾 & $x$ & $*$ & & $x \times x$ & $x$ & $\bar{x}$ & $1 / x$ & $\times$ \\
\hline & $x$ & & & $x \times$ & $x$ & $x$ & & $x$ & $x$ & $\square$ & $\times x$ & $x$ & $x$ & & & & & $x \times$ & $x$ & & m & 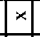 & $x$ & $x$ & $x \times x$ & of & & $x$ & \\
\hline & $\times \times \mid$ & $\star \times$ & & & $x$ & & $x$ & & $x \times$ & $\star x$ & $x$ & & $x$ & $\times \times$ & & & & $\star$ & 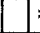 & & ले & $\times$ & & $x$ & $x \times 1$ & $x$ & & $x$ & \\
\hline & $x$ & & $\times$ & & $\times$ & & $x$ & & & $\times$ & & $\times$ & $x$ & $\times$ & & & & $\stackrel{x}{x}$ & & & 政 & & $\times$ & E & $\mid \times x$ & 4 & 1 & 11 & \\
\hline & $\times \times$ & & & $x \times$ & & $\times$ & & $x$ & $\times$ & $\star x$ & $\times x$ & $x$ & $x$ & & & $\star$ & & $\star$ & & & लㄹ & & $*$ & & $x \times$ & & & $x$ & \\
\hline & & & & $x$ & $\times$ & & $x$ & & $\star x$ & \begin{tabular}{|l|} 
\\
\end{tabular} & $\times$ & $\star$ & $\times$ & & & & & $\stackrel{\star}{*}$ & & & సิ & & 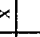 & & $\star$ & $x=$ & $\times$ & $\square$ & \\
\hline & & $x$ & & & $x$ & & & $x$ & & $x$ & 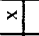 & & $x$ & & & & & $\times x$ & $x$ & $x$ & A2 & $x$ & & & $x \times$ & & & $|x| x$ & x) \\
\hline & & $x \times$ & $\times ;$ & $\times x$ & 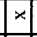 & & $x$ & & & $\times$ & $x \times$ & $x$ & $x$ & & $\times=$ & & & $\times$ & $x$ & & व & & $\times$ & & $1 \times$ & 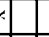 & & $|x| x$ & $\times$ \\
\hline & $x$ & $x$ & $x:$ & \begin{tabular}{l|l}
$x$ & $x$
\end{tabular} & $x$ & $x$ & & & 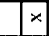 & & $x$ & $\times$ & $x$ & $\bar{x}$ & & $\bar{\star}$ & & $\bar{x} x$ & $\mid x$ & $\star x \mid$ & ल) & & $x$ & & $x$ & $x$ & & $|x| x$ & $x$ \\
\hline & 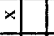 & $x$ & & $\underset{x}{x}$ & & & $\bar{x}$ & & \begin{tabular}{|c|}
$\times x$ \\
\end{tabular} & $\times$ & $x>$ & $\sqrt{x}$ & $x$ & & $x=$ & $\bar{x}$ & & $\bar{x}$ & $x$ & & (7) & & & $\bar{x}$ & 犬。 & f & $x$ & $x$ & \\
\hline & 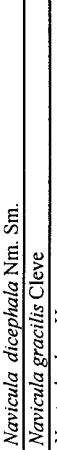 & & 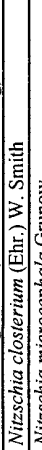 & : & : & & & & 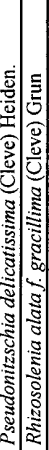 & $\mid$ & & בְב & 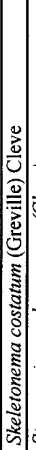 & 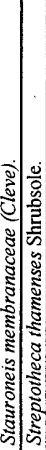 & 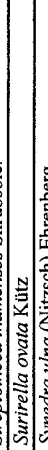 & 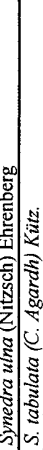 & | & & 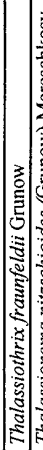 & : & 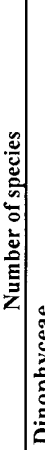 & 竎 & 章 & 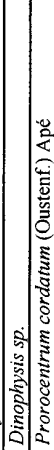 & : & 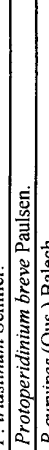 & 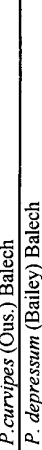 & 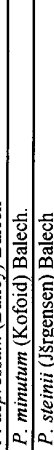 & 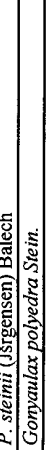 \\
\hline
\end{tabular}




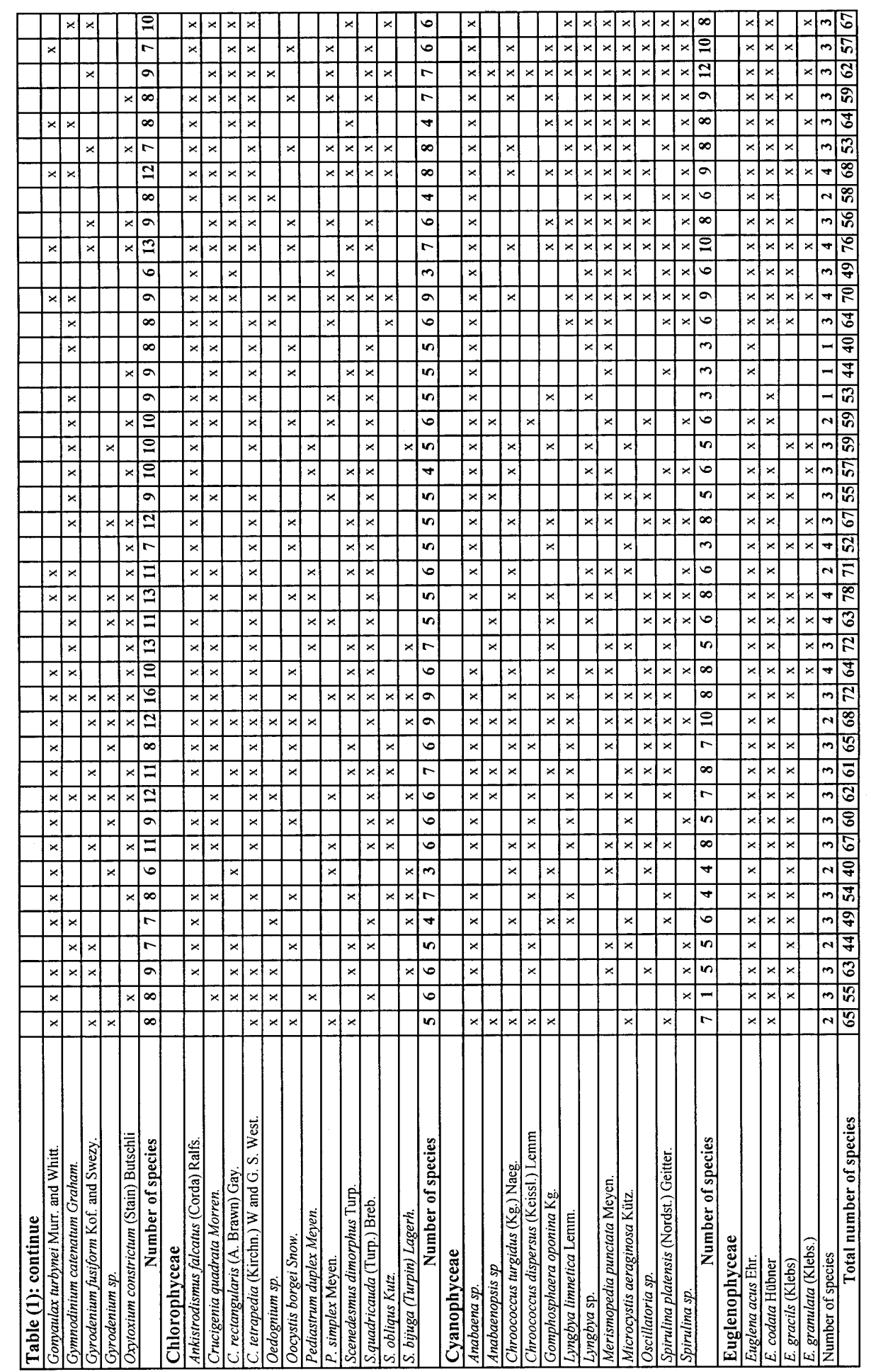


There were strong positive correlation between Skeletonema costatum and water temperature $(\mathrm{r}=0.54, \geq 0.05)$, while negative correlation for Asterionella spp $(\mathrm{r}=-0.40, \geq 0.05)$.

Abu-Qir Bay showed a marked variability of phytoplankton abundance in space and time as well as in community structure. The phytoplankton density (Figure 3) varied widely during the study period between $1737 \times 10^{3}$ unit. $\mathrm{L}^{-1}$ at St. 14 and $6616 \times 10^{3}$ unit. $L^{-1}$ near TPS (Table 3) with an average of $3595 \times 10^{3}$ unit. $^{-1}$ (Table 2).

Table (2): Average numbers of the different phytoplankton groups $\left(\mathrm{x}^{10} \mathrm{U}^{3} \mathrm{Unit}^{-1} \mathrm{~L}^{-1} \pm\right.$ standard deviation) and their percentage frequencies in Abu-Qir Bay during February, April and June 2004.

\begin{tabular}{|c|c|c|c|c|c|c|c|c|}
\hline \multirow{2}{*}{$\begin{array}{l}\text { Season } \\
\text { Groups }\end{array}$} & \multicolumn{2}{|c|}{ February } & \multicolumn{2}{|c|}{ April } & \multicolumn{2}{|c|}{ June } & \multirow[b]{2}{*}{ Average } & \multirow[b]{2}{*}{$\%$} \\
\hline & No. & $\%$ & No. & $\%$ & No. & $\%$ & & \\
\hline Bacillario. & $2365 \pm 1942$ & 90.86 & $2595 \pm 1022$ & 95.26 & $5269 \pm 1905$ & 95.18 & $3387 \pm 1827$ & 94.2 \\
\hline Dino. & $104 \pm 43$ & 4.00 & $58 \pm 14$ & 2.13 & $124 \pm 72$ & 2.24 & $93 \pm 50$ & 2.6 \\
\hline Chloro. & $35 \pm 17$ & 1.34 & $28 \pm 7$ & 1.03 & $37 \pm 18$ & 0.67 & $33 \pm 12$ & 0.9 \\
\hline Cyano. & $52 \pm 27$ & 2.00 & $21 \pm 5$ & 0.77 & $67 \pm 38$ & 1.21 & $47 \pm 30$ & 1.3 \\
\hline Eugleno. & $47 \pm 26$ & 1.80 & $22 \pm 12$ & 0.81 & $39 \pm 20$ & 0.70 & $36 \pm 19$ & 1.0 \\
\hline Total & $2603 \pm 1928$ & 100 & $2724 \pm 1032$ & 100 & $5536 \pm 2033$ & 100 & $3595 \pm 1862$ & 100 \\
\hline
\end{tabular}

During February, the phytoplankton counts showed the lowest density (Figure 3), they fluctuated between $934 \times 10^{3}$ unit/L (St. 14) and $6768 \times 10^{3}$ unit.L - $^{-1}$ (St.3) with an average of $2603 \times 10^{3}$ unit. $^{-1}$ (Tab. 2). As represented in table (1, 2), the community included 112 species, diatoms were the main bulk $(90.86 \%$ of the total phytoplankton count) with 69 species and predominated by Skeletonema costatum (51.3\%) and less extend Asterionella spp (8.7\%). The highest counts were recorded at the western part of the bay especially Sts. 1, 2 and 3 (Figure 4). On the other hand, diatoms Nitzchia spp. (1.7\%); Cyclotella spp. (2.7\%); Leptocylindrus danicus (2.1); Rhisosolenia delicatula (4.2\%) and Thalassiosera rotulla $(2.2 \%)$ were rarely recorded, in addition to dinophycean Prorocentrum spp. $(1.9 \%)$ and Protoperidenium spp., (1.6 \%). Freshwater forms were represented mainly by Cyanobacterium Merismopedia punctata which formed 0.8 $\%$ of the total phytoplankton especially at Sts. 1, 4, 8, 13 and 14. Euglena spp. was recorded at all stations with a percentage of $0.9 \%$ of the total phytoplankton (Figure 4).

During April, the highest phytoplankton density appeared near TPS and the counts of total phytoplankton decreased seaward (Figure 5). They fluctuated between $1172 \times 10^{3}$ unit. $\mathrm{L}^{-1}$ at St. 14 and $4073 \times 10^{3}$ unit.L $\mathrm{L}^{-1}$ at St. 3 with an average $2724 \times 10^{3}$ unit.L ${ }^{-1}$ (Fig. 5 and Tab. 2). The community included 99 species (Table1), in which diatoms contributed $95.26 \%$ (Table 2) of the total phytoplankton count (60 species) and represented mainly by Skeletonema 
costatum (41.3\%) and Nitzchia serriata $(46.5 \%)$. The two latter species formed a peak near TPS (Figure 5).

Table (3): Average counts of different phytoplankton groups ( $\left(\mathrm{x}^{3} \mathrm{Unit}^{3} \mathrm{~L}^{-1} \pm\right.$ standard deviation) at different stations in Abu-Qir Bay during the study period.

\begin{tabular}{|c|c|c|c|c|c|c|c|c|c|c|c|c|c|c|}
\hline \multirow{2}{*}{ 올 } & \multicolumn{14}{|c|}{ Stations } \\
\hline & 1 & 2 & 3 & 4 & 5 & 6 & 7 & 8 & 9 & 10 & 11 & 12 & 13 & 14 \\
\hline \multirow{3}{*}{ 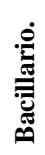 } & 4316 & 5118 & 6312 & 4058 & 4549 & 3114 & 2494 & 3956 & 2958 & 2819 & 2215 & 2130 & 1813 & 1565 \\
\hline & \pm & \pm & \pm & \pm & \pm & \pm & \pm & \pm & \pm & \pm & \pm & \pm & \pm & \pm \\
\hline & 1745 & 840 & 2267 & 2104 & 3323 & 1707 & 1871 & 2970 & 1283 & 1033 & 1019 & 870 & 1356 & 1188 \\
\hline \multirow{3}{*}{$\dot{\mathscr{g}}$} & 37 & 72 & 144 & 118 & 137 & 79 & 93 & 116 & 91 & 78 & 69 & 107 & 81 & 81 \\
\hline & \pm & \pm & \pm & \pm & \pm & \pm & \pm & \pm & \pm & \pm & \pm & \pm & \pm & \pm \\
\hline & 0 & 34 & 108 & 44 & 110 & 33 & 31 & 78 & 20 & 14 & 11 & 78 & 40 & 48 \\
\hline \multirow{3}{*}{ 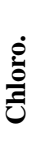 } & 22 & 49 & 37 & 28 & 44 & 37 & 18 & 41 & 29 & 32 & 35 & 27 & 32 & 29 \\
\hline & \pm & \pm & \pm & \pm & \pm & \pm & \pm & \pm & \pm & \pm & \pm & \pm & \pm & \pm \\
\hline & 1 & 26 & 21 & 8 & 35 & 18 & 3 & 8 & 10 & 5 & 7 & 3 & 16 & 7 \\
\hline \multirow{3}{*}{ 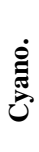 } & 56 & 29 & 70 & 33 & 71 & 35 & 39 & 63 & 35 & 38 & 41 & 37 & 63 & 43 \\
\hline & \pm & \pm & \pm & \pm & \pm & \pm & \pm & \pm & \pm & \pm & \pm & \pm & \pm & \pm \\
\hline & 32 & 24 & 54 & 14 & 78 & 15 & 23 & 41 & 8 & 14 & 17 & 25 & 53 & 21 \\
\hline \multirow{3}{*}{ 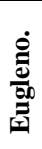 } & 38 & 42 & 53 & 39 & 70 & 34 & 20 & 37 & 35 & 30 & 29 & 20 & 24 & 18 \\
\hline & \pm & \pm & \pm & \pm & \pm & \pm & \pm & \pm & \pm & \pm & \pm & \pm & \pm & \pm \\
\hline & 25 & 29 & 32 & 10 & 47 & 20 & 11 & 18 & 26 & 4 & 15 & 6 & 12 & 11 \\
\hline \multirow{2}{*}{ 苞 } & 4469 & 5310 & 6616 & 4277 & 4871 & 3299 & 2664 & 4213 & 3148 & 2997 & 2389 & 2331 & 2012 & 1737 \\
\hline & $\begin{array}{c} \pm \\
1752\end{array}$ & $\begin{array}{c} \pm \\
1905\end{array}$ & $\begin{array}{c} \pm \\
2469\end{array}$ & $\begin{array}{c} \pm \\
2120\end{array}$ & $\stackrel{ \pm}{3530}$ & $\stackrel{ \pm}{1652}$ & $\begin{array}{c} \pm \\
1883\end{array}$ & $\begin{array}{c} \pm \\
3069\end{array}$ & $\stackrel{ \pm}{ \pm}$ & $\stackrel{ \pm}{1051}$ & $\begin{array}{c} \pm \\
1018\end{array}$ & $\begin{array}{c} \pm \\
1844\end{array}$ & $\stackrel{ \pm}{1335}$ & $\stackrel{ \pm}{ \pm}$ \\
\hline
\end{tabular}

The flourishing of both phytoplankton species and counts were observed during June (Table 1, 2). It fluctuated between $3105 \times 10^{3}$ unit.L ${ }^{-1}$ (St. 14) and $9004 \times 10^{3}$ unit.L ${ }^{-1}$ (St. 3) as shown in Figure (6) with an average of $5535 \times 10^{3}$ unit.L $\mathrm{L}^{-1}$ (Table 2). The community included 104 species (Table 1) where diatoms constituted the main bulk (64 species) and dominated by Skeletonema costatum (60.4\% of the total phytoplankton count). Nitzchia serriata; Rhizosolenia delicatula and Leptocylindrus danicus (10.6; 9.8 and $8.6 \%$, respectively) was frequently recorded (Figure 6). On the other hand Thalassiosera rotulla and Prorocentrum spp. (1.7 and 1.6\%) was rarely recorded (Figure 6).

The absolute values of species diversity index (Figure 7) displayed narrow temporal and spatial variations from 1.02 to 2.85 nats. Its average along 
the study period reported the lowest value (1.4 nats) in April and June and the highest (2.17 nats) in February. Throughout the bay, St. 3 sustained the lowest diversity index (1.38 nats), while St. 14 recorded the highest value (1.95 nats). The diversity index was negatively correlated with total phytoplankton and total Bacillariophyceae ( $r=-0.45$ and -0.49 , respectively $\geq 0.05$ ).

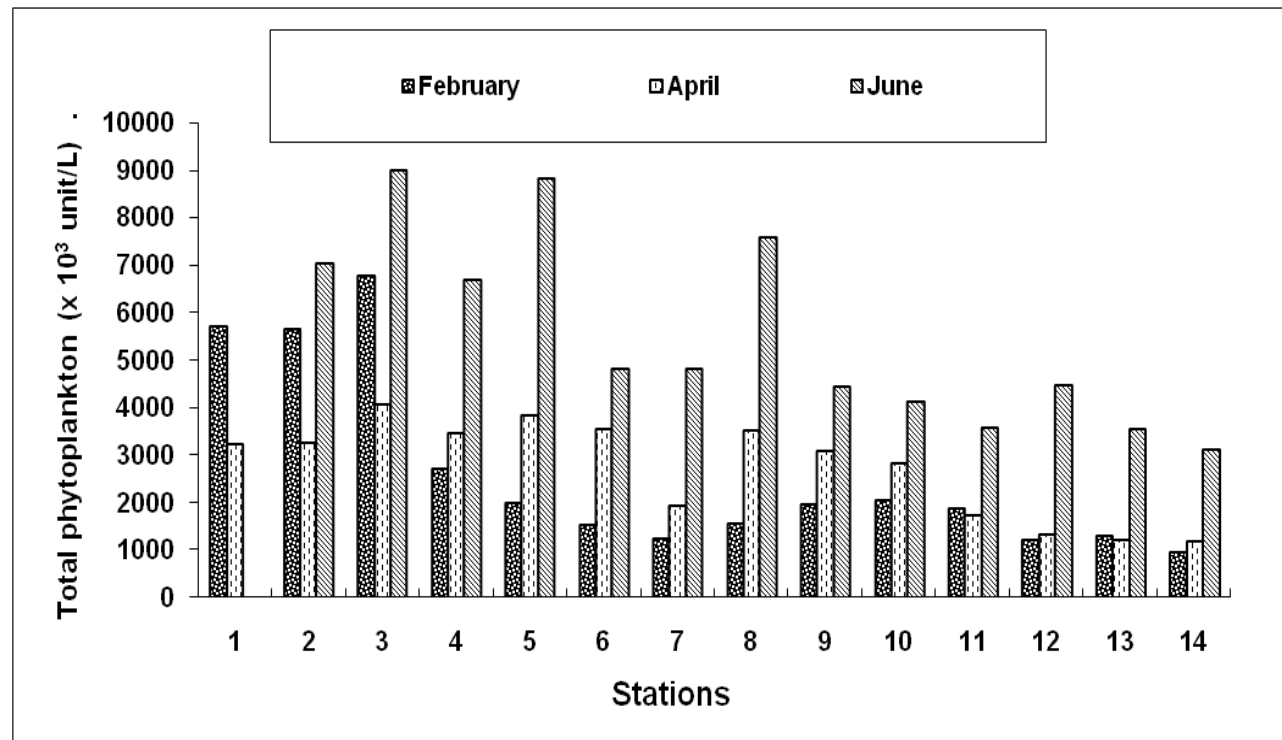

Figure (3): Variations of the total phytoplankton in Abu-Qir Bay at different stations during February during February, April and June 2004

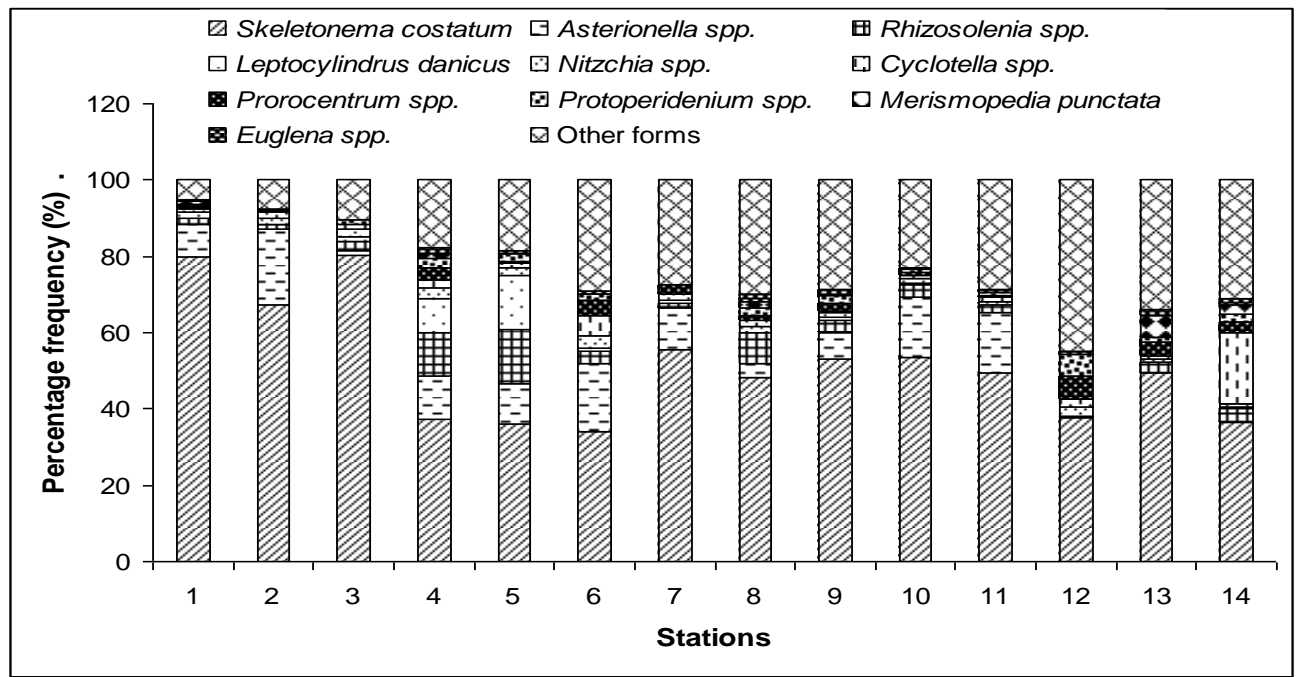

Figure (4): Percentage frequency of the main phytoplankton species at different stations in Abu-Qir Bay during February 2004 


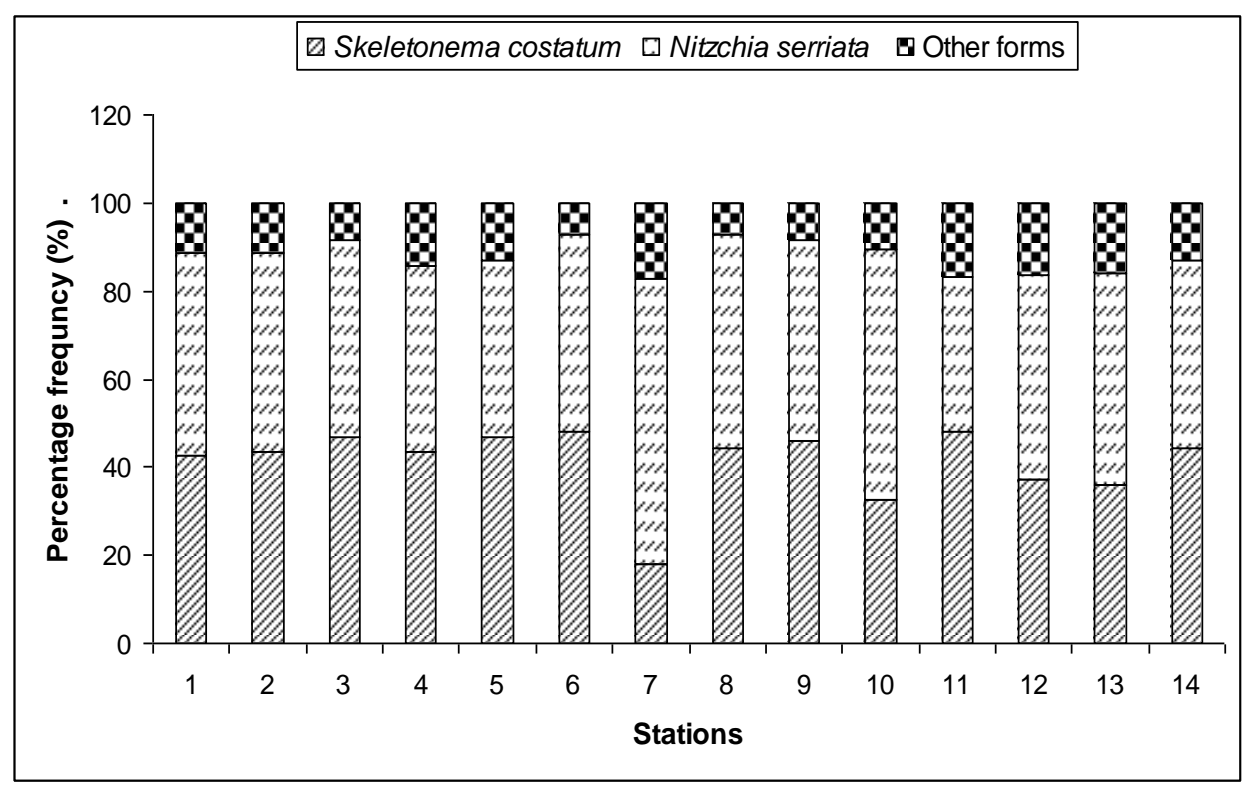

Figure (5): Percentage frequency of the main phytoplankton species at different stations in Abu-Qir Bay during April 2004

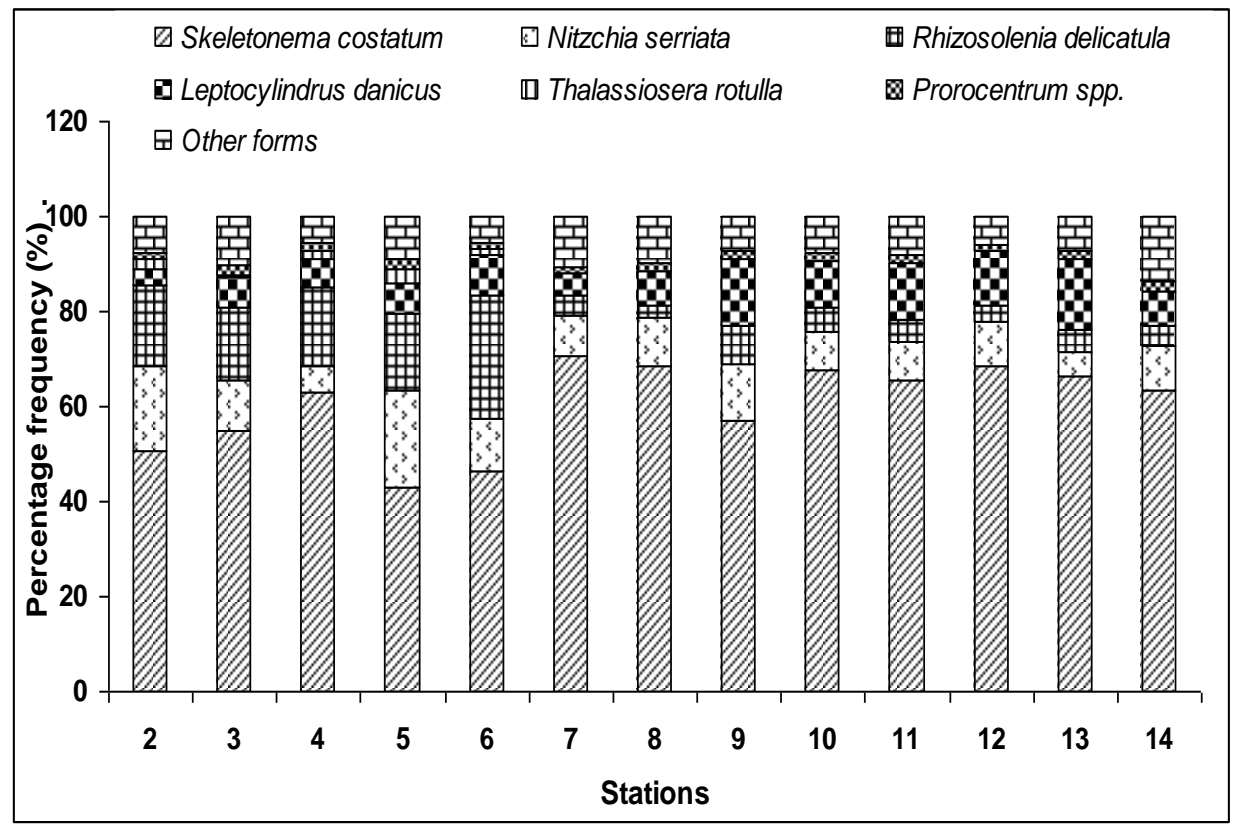

Figure (6): Percentage frequency of the main phytoplankton species at different stations in Abu-Qir Bay during June 2004 


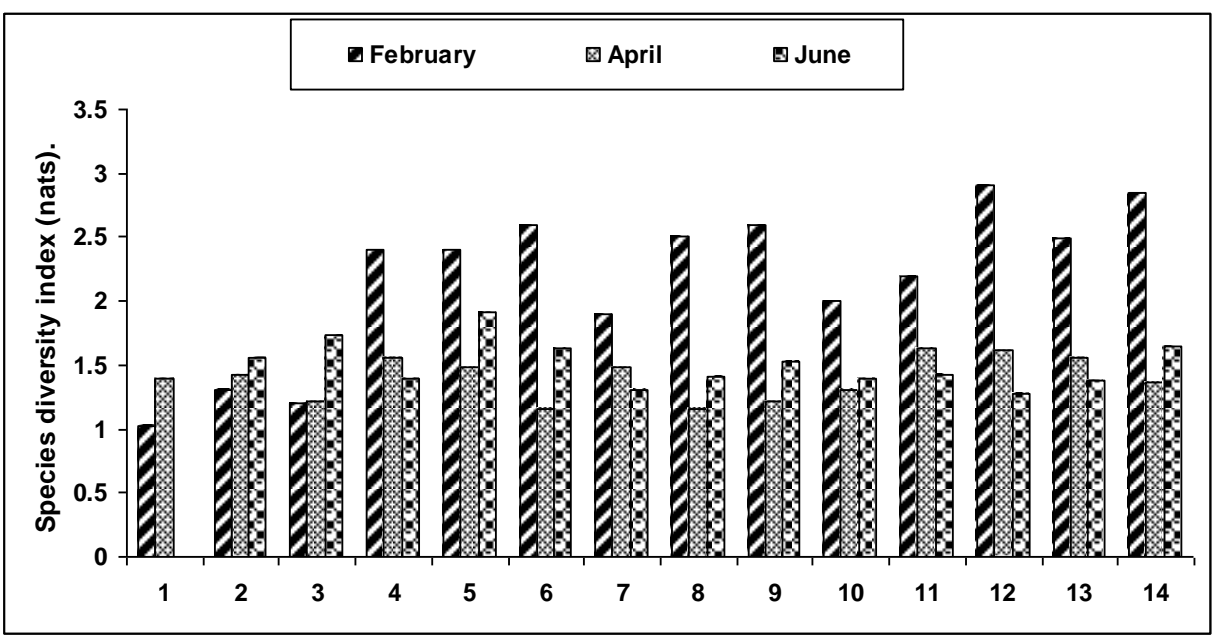

Figure (7): Species diversity index at different stations in Abu-Qir Bay during February, April and June 2004

\section{Discussion}

Semi-enclosed coastal systems, such as estuaries and bays have generally been regarded as one of the most productive aquatic systems in which nutrient supply is an important factor in sustaining the higher phytoplankton abundance and biomass (Bizsel et al., 2001). Abu-Qir Bay is considered one of the heavy polluted areas because it lies in the vicinity of discharged wastes and referred as a hot spot. In the present study, the high levels of nutrient salts (discussed and published by Aly-Eldeen, 2006) and high density of phytoplankton in addition to low dissolved oxygen concentrations $\left(<1.0 \mathrm{mg} \mathrm{O} \mathrm{O}_{2} \mathrm{~L}^{-1}\right)$, indicate an acute eutrophication in the bay. Water salinity values could be used as indicator of the flow of discharged wastes rich in nutrient salts which in turn reflect on the density and structure of the phytoplankton community throughout the bay. Stations 3-6 which are mainly subjected to the discharged water from Lake Edku and TPS usually showed high phytoplankton density, this mean that the discharge water promotes algal growth.

Throughout the last decade, dissolved inorganic nitrogen fluctuated from year to year, but dissolved inorganic phosphate showed a steady decrease, they were 23.27 and $1.42 \mu \mathrm{M}$ during 1998-1999 (Shams-El-Din and Dorgham, 2007) and during 2004 parallel to that studied was 19.64 and $0.68 \mu \mathrm{M}$ (Aly-Eldeen, 2006) for dissolved inorganic nitrogen and dissolved inorganic phosphate, respectively.

A total of 117 species of 5 classes were distinguished, Bacillariophyceae (>90\%) exhibited the widest range of maximum potential growth rates and occurred under a wide range of discharges. The freshwater groups Chlorophyceae, Cyanophyceae and Euglenophyceae appeared mainly at near 
shore stations, but usually with low percentage to the total phytoplankton count. Mohammed (1981) stated that, the current in Boughaz El-Maadiya is mostly directed seawards during spring and summer, but in winter it directed towards the Lake Edku. This observation is in agreement with the present results which showed that, the discharge waters covered most of the bay stations during June.

The phytoplankton count during the present study was markedly high (average $5535 \times 10^{3}$ unit. $\mathrm{L}^{-1}$ ). When compared it with previous records, the phytoplankton counts were $36 \times 10^{3}$ unit. $^{-1}$ (Samaan and Mikhail, 1990); 33 x $10^{3}$ unit. $L^{-1}$ by El-Sherif and Gharib (1994); $5 \times 10^{3}$ unit. $L^{-1}$ (Shams-El-Din and Dorgham, 2007) and $780 \times 10^{3}$ unit. $^{-1}$ (El-Sherif and Mikhail, 2003). Furthermore, the dominance of species was mostly different, Shams-El-Din and Dorgham (2007) reported species as Nitzchia pungens, Nitzchia pacifica, Asterionella japonica, Thalassionema nitzchoides, Chaetoceros affinis, Chaetoceros didymus, Prorocentrum micans and Euglena acus were the dominant species during 1998-1999. But during the next year 1999-2000 (El-Sherif and Mikhail, 2003) recorded other different species as, Asterionella glaciales, Skeletonema costatum, Rhizosolenia fragilissima, Leptocylindrus minimus, Chaetoceros affinis, Nitzchia longissima, Nitzchia serriata, Cyclotella meneghiniana, Cyclotella nana, Prorocentrum triestinum, Scripsiella trochoidea and Carteria cordiformis. During the present study, species as Skeletonema costatum, Nitzchia serriata, and Asterionella glaciales showed continuous dominancy. Furthermore, high counts of fresh and brackish water forms as Cyclotella meneghiniana, Scenedesmus spp., Nitzchia palea were recorded during 1997 in front of Boughaz El-Maadiya by Gharib and Dorgham (2000). None of these species appeared among the dominant species at the same area or at any other part of the bay during the present study.

The differences in the community composition between the present study and previous records may be attributed mainly to that the present study based on sampled collected in three months only, where the earlier study samples collected monthly all the year round. This was emphasized by the low number of species recorded in the present study (117 species) compared by 183 species (Shams-ElDin and Dorgham, 2007) and 241 species (El-Sherif and Mikhail, 2003). Such great increase in the phytoplankton density during the present study, the dominancy of few species and the decreased in dissolved oxygen reached $<1.0$ $\mathrm{mgO}_{2} \cdot \mathrm{I}^{-1}$ in some stations may be a serious sign to the eutrophication in the bay.

The present study indicated that Skeletonema costatum as the dominant form, it is considered as indicator for pollution (Mihnea, 1985) and this species was euryhaline and eurythermal, it known as indicator of eutrophication everywhere (Huang et al., 2004; Toming and Jaanus, 2007). Skeletonema costatum seem to respond quickly to new nutrients induced, known as fastgrowing diatoms (Malej et al., 2003) and previously recorded as dominant species in Abu-Qir Bay (Anonymous, 1981; Samaan and Mikhail, 1990; El-Sherif and Gharib, 1994). Asterionella glaciales and Skeletonema costatum are well known Egyptian J. of Phycol. Vol. 9, 2008 
red tide species, with occurrence closely related to land drainage (Revelante and Gilmartin, 1985; Labib, 1997). Asterionella glaciales reached $20 \times 10^{6}$ cell.. $\mathrm{L}^{-1}$ east of Boughaz El-Maadiya and $12 \times 10^{6}$ cell.. $\mathrm{L}^{-1}$ in front of TPS (Anonymous, 1981). Skeletonema costatum is a neritic diatom species with optimum salinity of $19.5 \mathrm{ppt}$ and declines as salinity deviates from this optimum (Cloern and Cheng, 1981). Skeletonema costatum is considered the most important species in Alexandria waters (Dowidar, 1965; El-Maghraby and Halim, 1965). Nitzschia serriata was recorded as dominant species beside Skeletonema costatum during summer. The members of the genus Nitzschia are highly eurythermic and flourished well at temperature ranging between $10^{\circ} \mathrm{C}$ and $27^{\circ} \mathrm{C}$ (Goldman, 1977). This explains the non-dependence of the genus growth on temperature variations. Nitzschia serriata, gives an indication for eutrophication (Rao and Mohanchand, 1988). The presence of Nitzschia serriata in the bay gives an indication for eutrophication (Revelante and Gilmartin, 1985).

A stepwise multiple regression equation applied to predicting the average levels of environmental parameters in water bodies which affects phytoplankton community, the regression equation showed that:

$$
\begin{aligned}
& \text { Total phytoplankton }=37325+363 \text { Temperature }-1108 \text { Salinity. } \\
& \text { Skeletonema costatum }=29525+233 \text { Temperature }-868 \text { Salinity. } \\
& \text { Asterionella glaciales }=3802+104 \text { Salinity }+46 \text { DO. } \\
& \text { Nitzschia serriata }=-7445+232 \text { Salinity }-145 \text { DO. }
\end{aligned}
$$

This equation illustrate that the studied environmental factors which affect the magnitude of phytoplankton standing crop and the dominant species.

In conclusion, the ecological conditions of Abu-Qir Bay are of great variability due to interaction of several factors, including discharged wastewater from El-Tabia pumping station; out let of Lake Edku and Rosetta mouth of the River Nile. This variability caused high eutrophication, increase in species diversity index and the dominancy of some species which as indicator for eutrophication.

\section{Acknowledgments}

The author acknowledges the PI of the plan (Prof. Dr. Mohamed Mamdouh Abbas) for his helpful cooperation, also I acknowledge Prof. Dr. Zeinab Mahmoud El-Sherif for her scientific helps.

\section{References}

Abdel-Aziz, N. E. M; Fahmy, M. A. and Dorgham, M. M. (2001). Hydrography, nutrient levels and plankton abundance in Abu-Qir Bay, Egypt. Medit. Marine Sci., 2 (2): 13 - 17.

Aly-Eldeen, M. M. A. E. (2006). Chemical studies on the interstitial water of Abu-Qir Bay of Alexandria (Egypt). M. Sc. Thesis, Fac. Sci. Al-Azhar Univ., 279 p.

Egyptian J. of Phycol. Vol. 9, 2008 
Anonymous (1981). Investigation of level and effects of pollutants in saline lakes littoral marine environments. Abu-Qir and Lake Edku. Academy of Science Research and Technology. Nat. Inst. Oceanog. \& Fish., Alex. 180pp.

Anonymous (1984). Pollution status of Abu-Qir Bay. Report, Nat. Inst. Oceanogr. \& Fish., 255pp.

Balech, E. (1948). Etude de quelques especes Peridenium souvent confondues. Hydro., 1 (4): 390-409.

Balech, E. (1980). On thecal morphology of dinoflagellates with special emphasis on circular and sulcal plates. Ancentrociene. Del. Mar. Y. Limnol. Univ. Nat. Auton. Mexico., 7 (1): 57-68.

Bizsel, N.; Benli, H. A.; Bizsel, K. C. and Metin, G. (2001). A sunoptic study on the phosphate and phytoplankton relationship in the hypereutropicated Izmir bay (Aegean Sea). Turk. J. Engin. Environ. Sci., 25: 89-99.

Cloern, J. E. and Cheng, R. T. (1981). Simulation model of Skeletonema costatum population dynamics in Northern Sea Francisco Bay, California Estuarine. Coastal and Shelf Science, 12: 83-100.

Dowidar, N. M. (1965). Distribution and ecology of marine plankton in Alexandria and surroundings. Ph.D. Thesis, Alex. Univ., 334 pp.

Dowidar, N. M.; Gergis, M.; El-Samra, M. I. and El-Deek, M. K. (1983). General review of the ecological conditions of Abu-Qir Bay. Unesco reports in marine sciences, 20: 124-133.

El-Deeb, M. K. Z. (1977). Hydrography and chemistry of Abu0Qir Bay. M. Sc. Thesis, Fac. Sci. Alex. Univ., 194pp.

EL-Maghraby, A. M. and Halim, Y. (1965). A quantitative and qualitative study of the plankton of Alexandria waters. Hydrobiol., 25 (1-2): 221238.

El-Rayes, O. A.; Saad, M. A. H. and El-Nady, F. E. (1993). Dispersion pattern of freshwater in Abu-Qir Bay. "Proc. $3^{\text {rd }}$ Int. Conf. Environ. Protection is a must" 13-15 April 1993, Alexandria. 103-109.

El-Sherif, Z. M. and Gharib, S. M. (1994). Phytoplankton production and composition in Abu-Qir Bay. Proc. $4^{\text {th }}$ Int. Conf "Environmental Protection in a must" 10 - 12 May 1994, Alexandria, 291 - 306.

El-Sherif, Z. M. and Mikhail, S. K. (2003). Phytoplankton dynamics in the southern part of Abu-Qir Bay, Alexandria, Egypt. Eg. J. Aqu. Biol. \& Fish., 7(1): 219 - 239.

Fahmy, M. A. (1997). Hydrochemistry and nutrients of Abu-Qir Bay during 1995. Bull. Fac. Sc., Alex. Univ., 37 (2): 171-186. 
Gharib, S. M. and Dorgham, M. M. (2000). Weekly structure and abundance of phytoplankton in Boughaz El-Maadiya, Egypt. Egypt. J. Aquat. Biol. \& Fish., 4 (2): 183 - 210.

Goldman, J. C. (1977). Biomass production in mass cultures of marine phytoplankton at varying temperature. J. Exp. Mar. Biol. Ecol., 27: 161169.

Heimann, K.; Roberts, K. R. and Wetherbee, R. (1995). Flagellar apparatus transformation and development in Prorocentrum micans and $P$. minimum (Dinophyceae). Phycologia., 34 (4): 323-335.

Huang, L.; Jian, W.; Song, X.; Huang, X.; Liu, S.; Qian, P.; Yin, K.; and Wu, M. (2004). "Species diversity and distribution for phytoplankton of the Pearl River estuary during rainy and dry season," Mar. Poll. Bull., 49: 30 $-39$.

Krammer, K. and Lange-Bertalot, H. (1986). Süsswasserflora Von Metteleuropa. Bacillariophyceae. Part I: Naviculaceae. Edit by H. Ettl, et al., G. Fischer, 211: 876pp.

Labib, W. (1997). Eutrophication in Mex Bay, Alexandria (Egypt). Environmental studies and statistical approach. Bull. Nat. Inst. Oceanogr. \& Fish., A. R. E., 23 : 49 -68.

Malej, A.; Mozedic, P.; Turk, V.; Terzic, S.; Ahel, M. and Cauwet, G. (2003). Changes in particulate and dissolved organic matter in nutrient-enriched enclosures from an area influenced by mucilage: the northern Adriatic Sea. J. Plankton Res., 25 (8): 949-966.

Mihnea, P. E. (1985). Effect of pollution on phytoplankton species. Rapp. Comm. Int. Mer. Medit., 29 (9): 85 - 88.

Mohamed, E. E. (1981). Exchange of water masses between Lake Edku and Abu-Qir Bay. M Sc. Thesis, Fac. Sci. Alex. Univ, Egypt, 129p.

Mohamed, L.A. and El-Maradny, A. (2001). Studies on the water quality of Abu Qir Bay during December 1999 to November 2000. Proceeding of the $2^{\text {nd }}$ International conference and Exhibition for Life and Environment, 3-5 April, 2001, Alex., Egypt, 361-383.

Pankow, H. (1976). Algenflora der Ostsee. II. Plankton (Einschl. Bentischer Kieselalgen). -Jena. 493pp.

Rao, M. U. and Mohanchand, V. (1988). Water quality characteristics and phytoplankton of polluted Visakhapatnam Harbour. Mar. Environ. Res., (25): 23-43.

Revelante, N. and Gilmartin, M. (1985). Possible phytoplankton species as indicators of eutrophication in the Northern Adriatic Sea. Rapp. Comm. Int. Mer. Medit., 29 (9): 89-91. 
Richardson, K. and Jórgensen, B. B. (1996). Eutrophication: definition, history and effects. In: Jǿrgensen, B.B. and Richardson, K., (eds). Eutrophication in coastal marine ecosystems. Coastal Estuarine Studies, 52: 1 - 19

Said, M. A. (1991). A review of the water circulation in Abu-Qir Bay and its effect on pollution transport. Maritime Res. J., 16: 53 - 61 .

Said, M. A., Ennet, P. Kokkila, T. and Sarkula, J. (1995). Modelling of transport processes in Abu-Qir Bay, Alexandria (Egypt). Proceedings of the Second International Conference on the Mediterranean Coastal Environment, MEDCOAST 95, Tarragona, Spain, 1673-1687.

Samaan, A.A. and Mikhail, S. K. (1990). Distribution of phytoplankton in AbuQir bay (Egypt). Bull. Nat. Inst. Oceanogr. \& Fish., A. R. E., 16 (1): 6573.

Shams-El-Din, N. G. and Dorgham, M. M. (2007). Phytoplankton community in Abu-Qir Bay as a hot spot on the Southeastern Medeterranean Coast. Egypt. Egyp. J. Aqua. Res., 33 (1): 163 - 182

Shannon, G. E. and Weaver, W. (1963). "The mathematical theory of communication" Univ. of Illiois Press. Urbana,USA, 111 - 125.

Tayel, F. T. R. (1992). The physical and chemical conditions of Abu-Qir Bay waters, Alexandria, Egypt. Bull. High Inst. Public Health, 22 (1): 87-99.

Toming, K. and Jaanus, A. (2007). Selecting potential summer phytoplankton eutrophication indicator species for the northern Baltic Sea. Proc. Estonian Acad. Sci. Biol. Ecol., 65 (4): 297-311.

Utermöhl, H. (1958). Zur Vervollkommnung der quantitativen phytoplanktonMethodik. Mitt. Int. Ver. Theor. Angew. Limnol., 9: 1 -38. 


\title{
توزيع الهائمات النباتيه فى منطقه الجنوب الغربى للأسكندريه

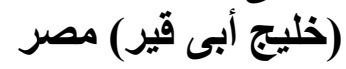

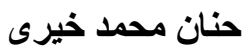 \\ المعهل القومى لعلوم البحار و الدصايد بالأسكندريه
}

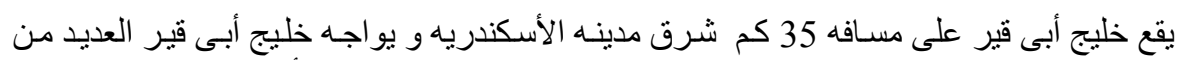

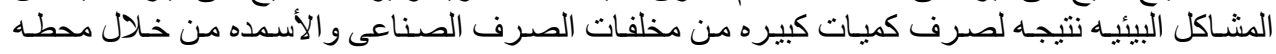

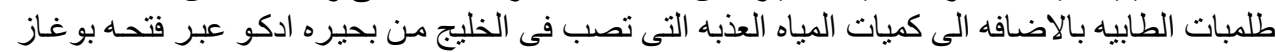

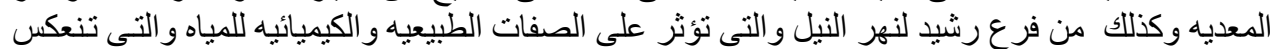
على الأحياء البحريه في خليج أبى قيلير. قد تم جمع عينات للهائمات النباتيه من 14 محطه تمثل البيئات المختلفه بخليج أبى قير خلال الفتره

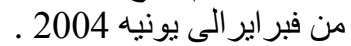

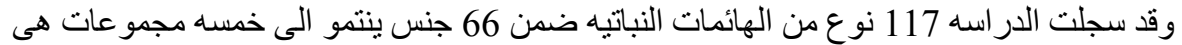

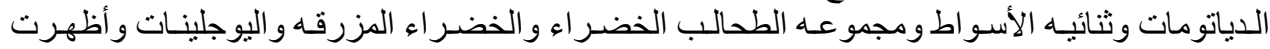

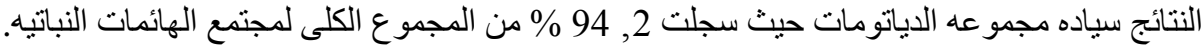

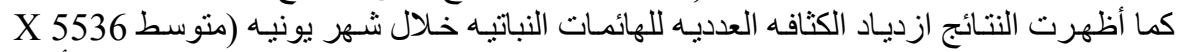
103 وحده لكل لتر) ) وكانت المحطات القريبه من محطه طلمبات الطابيه (محطه ،2، ،3، 4 ) هم الأكثر 